\title{
A Heuristic Approach to Fast NOVCA (Near Optimal Vertex Cover Algorithm)
}

\author{
Sanjaya Gajurel and Roger Bielefeld \\ ITS, Advanced Research ComputingCWRU, Cleveland 44106, USA
}

\begin{abstract}
This paper describes an extremely fast polynomial time algorithm, the NOVCA (Near Optimal Vertex Cover Algorithm) that produces an optimal or near optimal vertex cover for any known undirected graph G (V, E). NOVCA is based on the idea of (1) including the vertex having maximum degree in the vertex cover and (2) rendering the degree of a vertex to zero by including all its adjacent vertices. The three versions of algorithm, NOVCA-I, NOVCA-II, and NOVCA-random, have been developed. The results identifying bounds on the size of the minimum vertex cover as well as polynomial complexity of algorithm are given with experimental verification. Future research efforts will be directed at tuning the algorithm and providing proof for better approximation ratio with NOVCA compared to any available vertex cover algorithms.
\end{abstract}

Key words: Vertex cover problem, combinatorial problem, NP-complete problem, approximation algorithm, optimization, algorithms.

\section{Introduction}

The VC (vertex cover) of a graph $\mathrm{G}(\mathrm{V}, \mathrm{E})$ with vertex set $\mathrm{V}$ and edge set $\mathrm{E}$ is a subset of vertices $\mathrm{C}$ of $\mathrm{V}(\mathrm{C} \subseteq \mathrm{V})$ such that every edge of $\mathrm{G}$ has at least one endpoint in C. In 1972, Richard [1] showed that identification of minimal $\mathrm{VC}$ in a graph is an NP-complete problem.

Various algorithmic approaches have been used to tackle NP-complete problems. The vertex cover problem has been actively studied because of its important research and application implications. Polynomial-time approximation and heuristic algorithms for $\mathrm{VC}$ have been developed but none of them guarantee optimality. By using the definition of approximation ratio, $\mathrm{VC}$ has an approximation ratio of $\rho(n)$ for any input of size $n$. The solution $C$ produced by approximation algorithm is within the factor of $\rho(n)$ of the solution $\mathrm{c}^{*}$ of an optimal algorithm, i.e., $\mathrm{C}^{*} / \mathrm{C}$ $\leq \rho(n)$. Also, the approximation algorithm has approximation ratio of $2-\varepsilon$, where $0<\varepsilon<1$. A

Corresponding author: Sanjaya Gajurel, Ph.D., research fields: HPC, MANET, swarm algorithm, optimization. E-mail: sxg125@case.edu. 2-approximation [2] algorithm has been trivially obtained and similar approximation algorithms have been developed $[3,4]$ with an approximation of $(2-$ $(\ln (\ln n) / 2 \ln n))$, where $n$ is the number of vertices. Halperin [5] achieved an approximation factor of (2 $(1-\mathrm{o}(1))(2 \ln (\ln \Delta) / \ln \Delta))$ with maximum degree at most $\Delta$. Karakostas [6] attained an approximation factor of $(2-\theta(1 /(\log n) 1 / 2)))$, the best approximation yet, by using the semidefinite programming relaxation of VC. EA (evolutionary algorithms) that are randomized search heuristics have also been used for solving combinatorial optimization problems including $\mathrm{VC}[7,8]$.

Vertex cover problems have been solved in $\mathrm{O}$ $(1.2738 k+k n)$ time [9] by using a bounded search technique where a function of a parameter restricts the search space. Abu-Khazm et al. [10] have identified crown structure to reduce the size of both $n$ and $k$. It has been known that when relevant parameters are fixed, NP-complete problems can be solved in polynomial time. In both Ref. [10] and Ref. [11], $n$ is the input size and $k$ is the positive integer parameter. Though not guaranteed to find a minimum vertex 
cover, an approximation of $3 / 2$ for almost every single graph was obtained in Ref. [11]. According to Ref. [12], it is NP-hard to get $\varepsilon<1.3606$.

The paper is organized as follows: the NOVCA algorithm is described in Section 2; Section 3 provides experimental results; Section 4 is the conclusion.

\section{Near Optimal Vertex Cover Algorithm}

NOVCA is motivated by the fact that vertex cover candidates are those that are adjacent to minimum degree vertex so that its degree will be forcibly rendered to zero without choosing it. This fact has been reinforced during tie when the vertex with neighbors having maximum degrees is preferred over other minimum vertices. Without any optimization effort, the complexity of NOVCA is $\mathrm{O}\left(\mathrm{E}\left(V+\log ^{2} V\right)\right)$; with $\mathrm{V}=n$, the complexity becomes $\mathrm{O}\left(n^{2}\left(n+\log ^{2} n\right)\right)$ which is polynomial. Network Bench Node Degree algorithm [13] has been applied to determine the degree of each node. Then, the sum of the degree of adjacent nodes for each node is calculated. Both these values are included as data structures in a node $\operatorname{deg}[v] / \operatorname{adj} \_d e g \_s u m[v]$ as showed in pseudo-code of NOVCA algorithm as follows.

Input: $V$ is the set of vertices of $G, E$ is the set of edges of $G$, deg[V] is an integer array indexed by $V$ for a set of vertices $V$, sum_adj_deg[V] is an integer array indexed by $V$ for a set of vertices $V$, Qsum_adj_deg is the set of vertices having min $\operatorname{deg}[V]$.

Output: $V C$ is the set of vertices comprising a vertex cover.

Functions: Degree(v) is the degree of the vertex $v e$ $V, \operatorname{Adj}(v)$ gives the set of vertices that are adjacent to $v$ $\epsilon \quad V$, GetMinVertex() identifies the next adjacent vertices to include in the cover, Heap_MIN(deg) returns the value of min. $\operatorname{deg}[V]$, HEAP_MAX(Qsum_adj_deg) returns the vertex having max Qsum_adj_deg

for each $v \in V\{$

$\operatorname{deg}[v]=\operatorname{Degree}(v)$

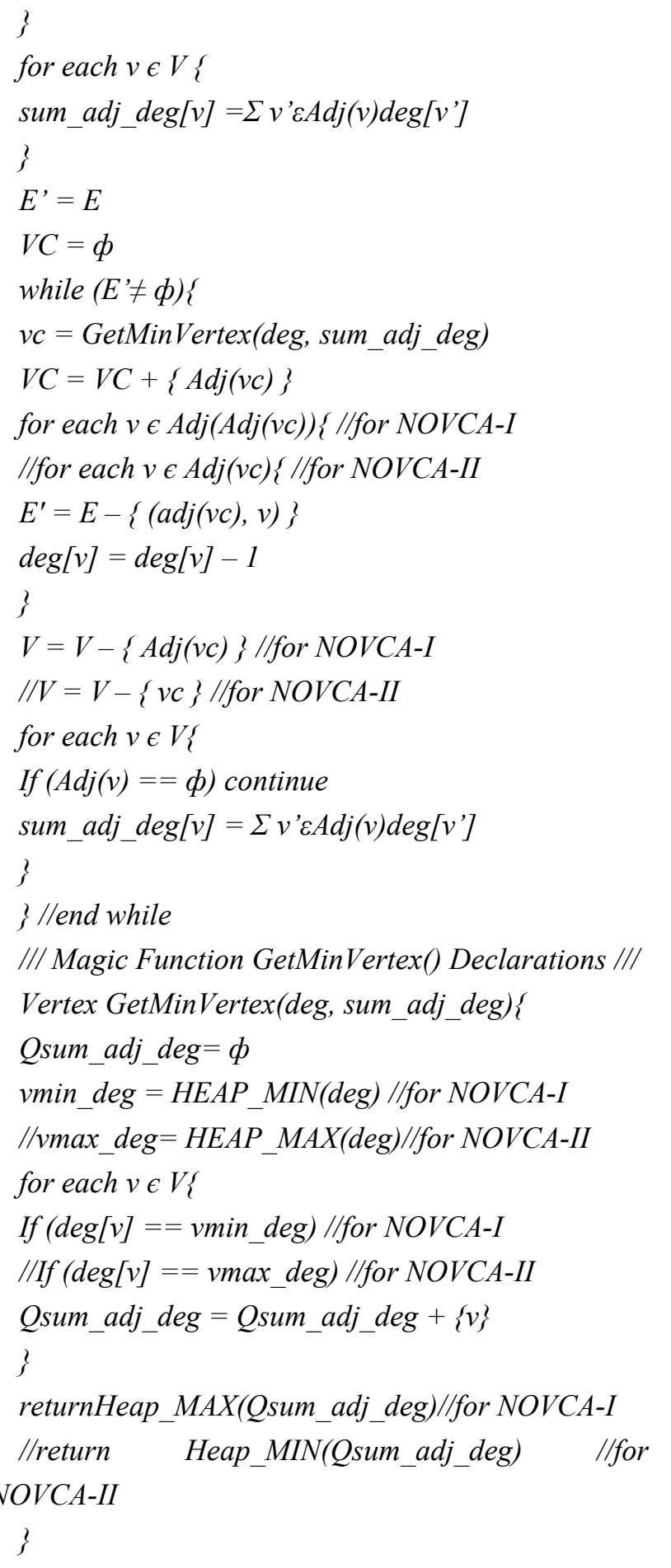

Initially, vertex cover set VC is empty. NOVCA-I [14] constructs the vertex cover by repeatedly adding, at each step, all vertices adjacent to the vertex of minimal degree. In the case of a tie, it chooses the one having the maximum sum of degrees of its neighbors. NOVCA-II [15], on the other hand, builds vertex 
cover by including vertices in descending order of degree and in the case of a draw; it selects the vertex having the minimum sum of degrees of its neighbors. The magic function GetMinVertex breaks a tie in selecting the best candidate vertex in a vertex cover. The implementation forcibly renders the degree of low degree vertices to zero without choosing them.

The version, NOVCA-random, selects NOVCA-I and NOVCA-II based on the random value, i.e., NOVCA-I and NOVCA-II are chosen with the probability of $85 \%$ and $15 \%$ respectively during each RUN as NOVCA has showed better performance in most of the instances.

\section{Experiment Works and Results}

Experiments to corroborate the theoretical results have been conducted on the CWRU HPC (high performance computing) resource using compute nodes with $3.0 \mathrm{GHz}$ Intel Xeon processors running Red Hat Enterprise Linux 4 and using the gec 3.4.6 compiler. We have selected complete graph as a test graph to determine time complexity of NOVCA for two reasons:

- Optimal vertex cover is known, $n-1$, where $n$ is the number of vertices;

- requires exhaustive search; there is an edge from each vertex to all other vertices.

The shell script "graph_gen.sh" generates a complete graph of size $n$ entered as input. This graph is then fed to executable "vc" $(\mathrm{C}++$ program compiled with $\mathrm{g}++$ compiler) to get vertex cover for that particular graph.

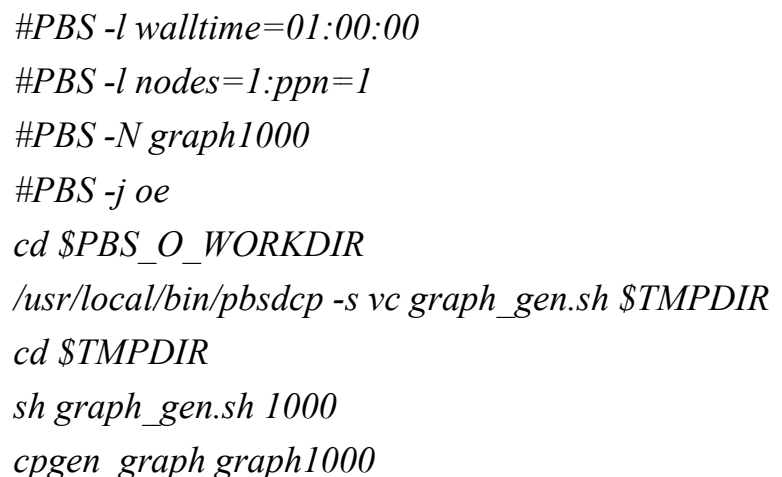

\#PBS -l nodes $=1: p p n=1$

\#PBS -N graph1000

\#PBS -j oe

cd \$PBS_O_WORKDIR

/usr/local/bin/pbsdcp -s ve graph gen.sh \$TMPDIR

sh graph_gen.sh 1000

cpgen_graph graph1000

time ./ve graph1000

/usr/local/bin/pbsdcp -g '*'\$PBS_O_WORKDIR cd \$PBS O WORKDIR

We have recorded the computation time for different sizes of the graphs to elucidate the polynomial complexity of NOVCA algorithm through MATLAB's polyfit $(x, y, n)$ command as showed in Figs. 1and 2.

NOVCA has approximation ratio smaller than 1.3606 for all available bench mark (Table 1, Table 2) and Table 3 [16]; all instances, however, are not showed here. For some instances like c-fat, Johnson, and random graphs NOVCA provides optimal cover. Noticeably, the execution time of NOVCA for any instance is remarkable. NOVCA has been found to perform very well compared to other available algorithms. For the instances where it provides near optimal solutions, it outperforms other algorithms in

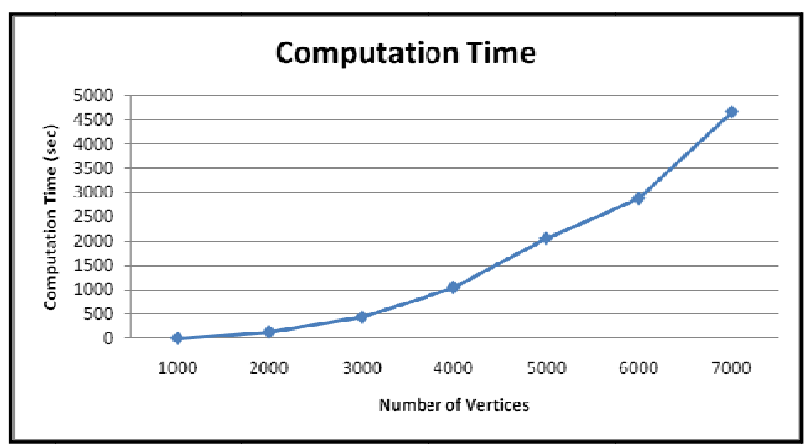

Fig. 1 Computational time of NOVCA for different sizes of complete graphs.

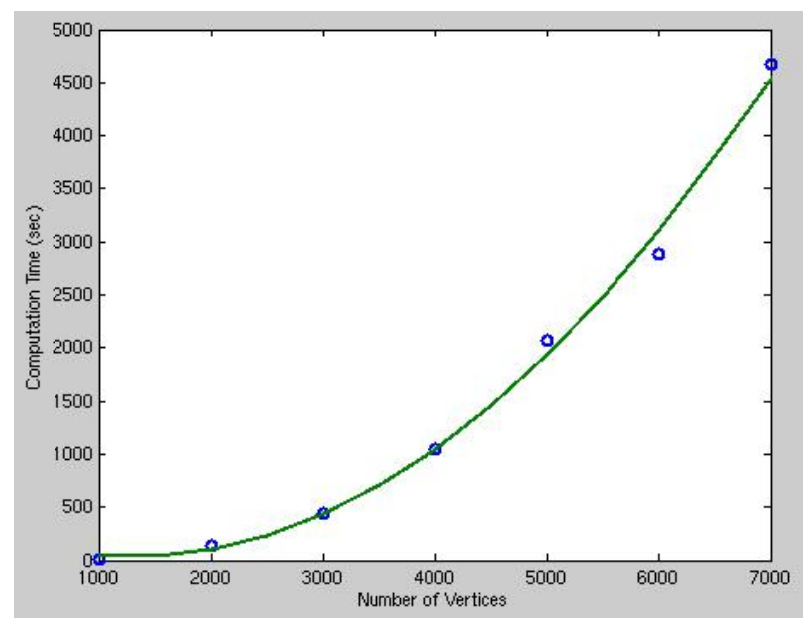

Fig. 2 MATLAB plot using polyfit with $n=2$. 
Table 1 DIMACS and BHOSLIB benchmarks.

\begin{tabular}{lll}
\hline Instances & $|V|$ & $\left|C^{*}\right|$ \\
\hline frb59-26-1 & 1,534 & 1,475 \\
frb59-26-2 & 1,534 & 1,475 \\
frb100-40 & 4,000 & 3,900 \\
broc200_1 & 200 & 179 \\
broc800_4 & 800 & 774 \\
C2000.9 & 2,000 & 1,922 \\
c-fat200-5 & 200 & 142 \\
c-fat500-10 & 500 & 374 \\
gen200_p0.9_44 & 200 & 156 \\
hamming10-2 & 1,024 & 512 \\
hamming10-4 & 1,024 & 984 \\
johnson16-2-4 & 120 & 112 \\
johnson32-2-4 & 496 & 480 \\
keller4 & 171 & 160 \\
keller5 & 776 & 749 \\
MANN_a27 & 378 & 252 \\
MANN_a81 & 3,321 & 2,221 \\
p_hat500-1 & 500 & 491 \\
p_hat1500-3 & 1,500 & 1,406 \\
san200_0.7_1 & 200 & 170 \\
san1000 & 1,000 & 985 \\
sanr200_0.7 & 200 & 183 \\
sanr400_0.7 & 400 & 379 \\
graph50-10 & 50 & 35 \\
graph50-10 & 50 & 35 \\
graph100-10 & 100 & 70 \\
graph200-05 & 200 & 150 \\
graph250-05 & 250 & 200 \\
graph500-05 & 500 & 290 \\
\hline & & \\
\hline
\end{tabular}

terms of execution time. We have compared NOVCA with COVER [17]. COVER is a stochastic local search algorithm for k-vertex cover. It constructs the initial candidate solution $C$ greedily. When the several vertices satisfy the criterion for inclusion in $C$, COVER selects one of them randomly with uniform probabilities. The COVER algorithm terminates when either the vertex cover is found or max number of steps (MAX_ITERATIONS) has been reached. NOVCA-I and NOVCA-II, on the other hand, do not have any randomness element and terminate when there are no more vertices in $V$. So, they have only one run unlike average execution time calculated using random seeds in different runs in COVER. NOVCA-random has randomness only in selection of two algorithmic approaches.

Though COVER is found to obtain better vertex cover in most of the instances of the benchmarks, NOVCA is very simple and it outperforms COVER in execution time. In case of the graph instance, MANN_a81, where both NOVCA and COVER return the same value 2,225, NOVCA is 20 times faster. Though NOVCA-I outperforms NOVCA-II in terms of approximation ratio in almost all instances except keller, p-hat, and sanr, NOVCA-II has better execution

Table 2 Performance comparison between NOVCA-I and COVER on DIMACS and BHOSLIB benchmarks $|V|$ : number of vertices; $\left|C^{*}\right|$ : optimal cover; NOVCA $|C|$ : cover returned by NOVCA; COVER $|C|$ avg: cover returned by COVER; NOVCA Time (s): execution time for NOVCA; COVER Time ${ }_{\text {avg: }}$ average execution time for COVER; no data available for the instance frb100-40 in COVER.

\begin{tabular}{|c|c|c|c|c|}
\hline $\begin{array}{l}\text { NOVCA-I } \\
|C|\end{array}$ & $\begin{array}{l}\text { NOVCA-I } \\
|C| /\left|C^{*}\right|\end{array}$ & $\begin{array}{l}\text { NOVCA-I } \\
\text { Time (s) }\end{array}$ & $\begin{array}{l}\text { COVER } \\
|C|_{\text {avg }}\end{array}$ & $\begin{array}{l}\text { COVER } \\
\text { Time }_{\text {avg }}(\mathrm{sec})\end{array}$ \\
\hline 1,485 & 1.007 & 80.258 & 1,477 & $1,8611.3$ \\
\hline 1,484 & 1.006 & 79.297 & 1,478 & $1,8589.5$ \\
\hline 3,917 & 1.004 & 2013.667 & - & - \\
\hline 181 & 1.011 & 0.115 & 179 & 768.2 \\
\hline 782 & 1.010 & 10.832 & 775 & $4,051.2$ \\
\hline 1,932 & 1.005 & 207.060 & 1,922 & $21,489.7$ \\
\hline 142 & 1 & 0.092 & 142 & $1,549.1$ \\
\hline 374 & 1 & 2.117 & 374 & $4,401.2$ \\
\hline 163 & 1.045 & 0.092 & 156 & $1,543.6$ \\
\hline 512 & 1 & 10.297 & 512 & $2,412.2$ \\
\hline 988 & 1.004 & 21.505 & 986 & $3,457.6$ \\
\hline 112 & 1 & 0.076 & 112 & 297.9 \\
\hline 480 & 1 & 2.273 & 480 & $2,351.9$ \\
\hline 164 & 1.025 & 0.007 & 160 & 985.7 \\
\hline 761 & 1.016 & 9.125 & 749 & $2,364.9$ \\
\hline 253 & 1.004 & 0.493 & 252 & 756.3 \\
\hline 2,225 & 1.002 & 773.963 & 2,225 & $15,672.1$ \\
\hline 492 & 1.002 & 2.683 & 491 & $1,810.2$ \\
\hline 1,414 & 1.006 & 74.991 & 1,406 & $1,298.9$ \\
\hline 183 & 1.077 & 0.117 & 170 & 713.7 \\
\hline 991 & 1.006 & 22.901 & 989 & $4,972.8$ \\
\hline 185 & 1.011 & 0.857 & 183 & 788.2 \\
\hline 382 & 1.008 & 1.030 & 380 & 2112.5 \\
\hline 35 & 1 & 0.006 & 35 & 124.5 \\
\hline 70 & 1 & 0.034 & 70 & 205.3 \\
\hline 150 & 1 & 0.114 & 150 & 854.1 \\
\hline 200 & 1 & 0.300 & 200 & 988.5 \\
\hline 290 & 1 & 1.604 & 290 & $22,555.2$ \\
\hline 1,485 & 1.007 & 80.258 & 1,477 & $18,611.3$ \\
\hline
\end{tabular}


Table 3 Performance comparison between NOVCA-II and COVER on DIMACS and BHOSLIB benchmarks $|V|$ : number of vertices; $\left|C^{*}\right|$ : optimal cover; NOVCA $|C|$ : cover returned by NOVCA; COVER $|C|_{\text {avg: }}$ cover returned by COVER; NOVCA Time (s): execution time for NOVCA; COVER Time avg: $_{\text {average execution time for COVER; no }}$ data available for the instance frb100-40 in COVER.

\begin{tabular}{|c|c|c|c|c|}
\hline $\begin{array}{l}\text { NOVCA-II } \\
|C|\end{array}$ & $\begin{array}{l}\text { NOVCA-II } \\
|C| / C^{*} \mid\end{array}$ & $\begin{array}{l}\text { NOVCA-II } \\
\text { Time (s) }\end{array}$ & $\begin{array}{l}\text { COVER } \\
|C|_{\text {avg }}\end{array}$ & $\begin{array}{l}\text { COVER } \\
\text { Time }_{\text {avg }}(\mathrm{s})\end{array}$ \\
\hline 1,494 & 1.014 & 34.770 & 1,477 & $18,611.3$ \\
\hline 1,496 & 1.014 & 35.686 & 1,478 & $18,589.5$ \\
\hline 3,944 & 1.011 & 885.860 & - & - \\
\hline 182 & 1.017 & 1.316 & 179 & 768.2 \\
\hline 786 & 1.016 & 6.162 & 775 & $4,051.2$ \\
\hline 1,942 & 1.010 & 88.604 & 1,922 & $21,489.7$ \\
\hline 142 & 1 & 1.238 & 142 & $1,549.1$ \\
\hline 374 & 1 & 1.514 & 374 & $4,401.2$ \\
\hline 170 & 1.090 & 1.514 & 156 & $1,543.6$ \\
\hline 512 & 1 & 5.584 & 512 & $2,412.2$ \\
\hline 992 & 1.008 & 10.350 & 986 & $3,457.6$ \\
\hline 112 & 1 & 1.248 & 112 & 297.9 \\
\hline 480 & 1 & 2.245 & 480 & $2,351.9$ \\
\hline 162 & 1.013 & 1.500 & 160 & 985.7 \\
\hline 761 & 1.016 & 5.115 & 749 & $2,364.9$ \\
\hline 261 & 1.036 & 1.641 & 252 & 756.3 \\
\hline 2,241 & 1.009 & 297.236 & 2,225 & $15,672.1$ \\
\hline 492 & 1.002 & 2.595 & 491 & $1,810.2$ \\
\hline 1,412 & 1.004 & 34.535 & 1,406 & 1298.9 \\
\hline 185 & 1.088 & 1.535 & 170 & 713.7 \\
\hline 992 & 1.007 & 11.657 & 989 & $4,972.8$ \\
\hline 184 & 1.005 & 1.351 & 183 & 788.2 \\
\hline 384 & 1.013 & 1.947 & 380 & $2,112.5$ \\
\hline 35 & 1 & 1.667 & 35 & 124.5 \\
\hline 70 & 1 & 1.552 & 70 & 205.3 \\
\hline 150 & 1 & 1.523 & 150 & 854.1 \\
\hline 200 & 1 & 1.653 & 200 & 988.5 \\
\hline 290 & 1 & 2.366 & 290 & $22,555.2$ \\
\hline 1,494 & 1.014 & 34.770 & 1,477 & $18,611.3$ \\
\hline
\end{tabular}

time than NOVCA-I. NOVCA-random always returns better cover than both NOVCA-I and NOVCA-II. For the challenge instances of frb100-40 [16], NOVCA-I is off by just 17 vertices (NOVCA returns 3,917 vertices whereas the optimal vertex cover is 3,900 ), but the execution time is just remarkable, only 2,013.667 s. The challenge is stated as "Based on theoretical analysis and experimental results of smaller instances, I conjecture that in the next 20 years or more (from 2005), these two benchmarks cannot be
Table 4 Performance of NOVCA-random on DIMACS and BHOSLIB benchmarks $|V|$ : number of vertices; $\left|C^{*}\right|$ : optimal cover; NOVCA $|C|$ : cover returned by NOVCA-I and NOVCA-II (one RUN); $|C|_{\min }$ : minimum number of vertices returned by NOVCA-random out of 1,000 RUNS.

\begin{tabular}{|c|c|c|}
\hline $\begin{array}{l}\text { NOVCA-I } \\
|C| \\
\end{array}$ & $\begin{array}{l}\text { NOVCA-II } \\
|C|\end{array}$ & $\begin{array}{l}\text { NOVCA-random } \\
|C|_{\text {avg }}\end{array}$ \\
\hline 1,485 & 1,494 & 1,479 \\
\hline 1,484 & 1,496 & 1,479 \\
\hline 3,917 & 3,944 & 3,904 \\
\hline 181 & 182 & 180 \\
\hline 782 & 786 & 780 \\
\hline 1,932 & 1,942 & 1,932 \\
\hline 142 & 142 & 142 \\
\hline 374 & 374 & 374 \\
\hline 163 & 170 & 162 \\
\hline 512 & 512 & 512 \\
\hline 988 & 992 & 984 \\
\hline 112 & 112 & 112 \\
\hline 480 & 480 & 480 \\
\hline 164 & 162 & 162 \\
\hline 761 & 761 & 754 \\
\hline 253 & 261 & 253 \\
\hline 2,225 & 2,241 & 2,221 \\
\hline 492 & 492 & 491 \\
\hline 1,414 & 1,412 & 1,408 \\
\hline 183 & 185 & 183 \\
\hline 991 & 992 & 990 \\
\hline 185 & 184 & 183 \\
\hline 382 & 384 & 380 \\
\hline 35 & 35 & 35 \\
\hline 70 & 70 & 70 \\
\hline 150 & 150 & 150 \\
\hline 200 & 200 & 200 \\
\hline 290 & 290 & 290 \\
\hline 1,485 & 1,494 & 1,479 \\
\hline
\end{tabular}

solved on a PC (or alike) in a reasonable time (e.g., 1 day) [16]." The graphs for number of vertices returned and the execution times, as showed in Figs. 3 and 4 respectively, portray that NOVCA, though comparable to COVER in terms of number of vertices returned, is significantly faster than COVER.

Table 4 compares all the versions of NOVCA which are then represented in bar diagrams in Fig. 5 to visualize the better performance of NOVCA-random compared to the earlier versions, NOVCA-I and NOVCA-II, based on the minimum number of vertices obtained from 1,000 runs. For the instance 
frb100-40, the minimum vertex cover returned by NOVCA-random $\left(|C|_{\min }=3,904\right)$ is considerably smaller than the covers returned by NOVCA-I $(|\mathrm{C}|=$ $3,917)$ and NOVCA-II $(|C|=3,944)$. We have also carried out comparisons of NOVCA against two other heuristic MVC (minimum vertex cover) algorithms, PLS [18] and EWCC [19], with similar results (not explicitly tabulated here).

\section{Vertex Cover}

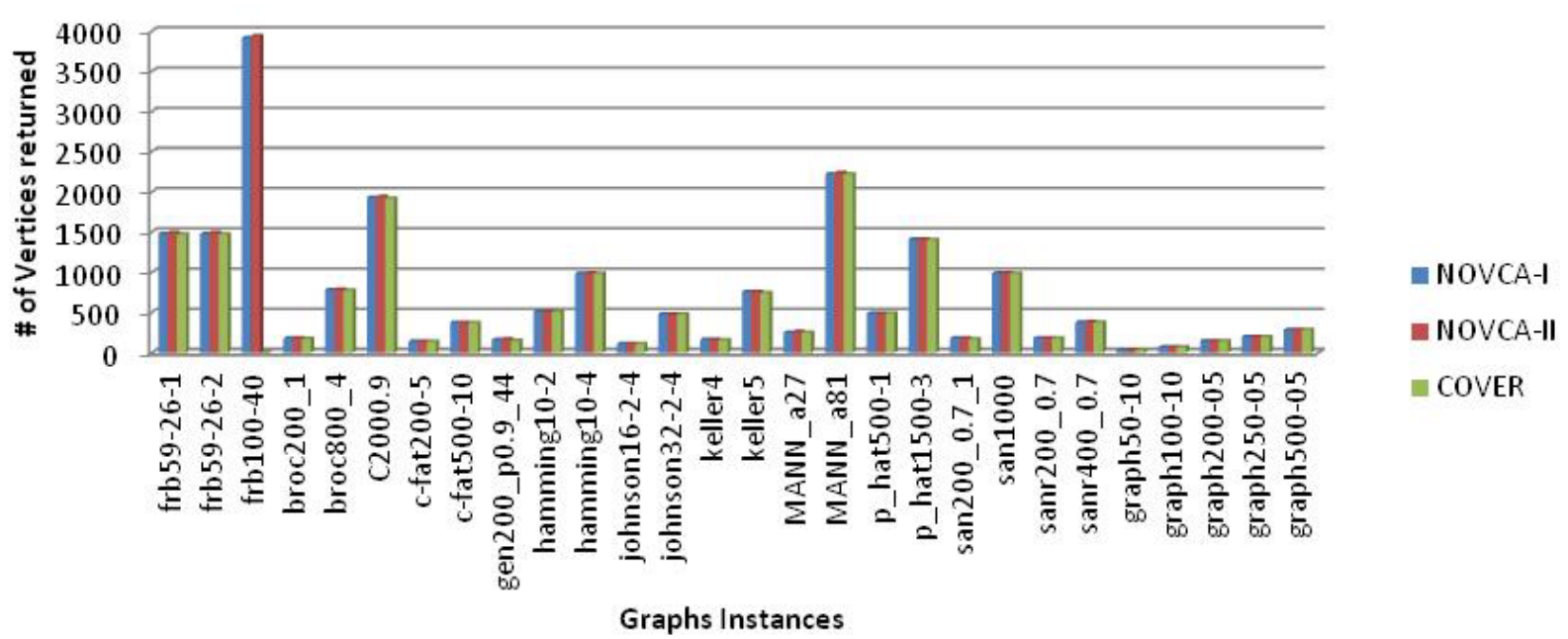

Fig. 3 Number of vertices returned by NOVCA-I, NOVCA-II, and COVER; no results from COVER for the instance frb100-40.

\section{Execution Time}

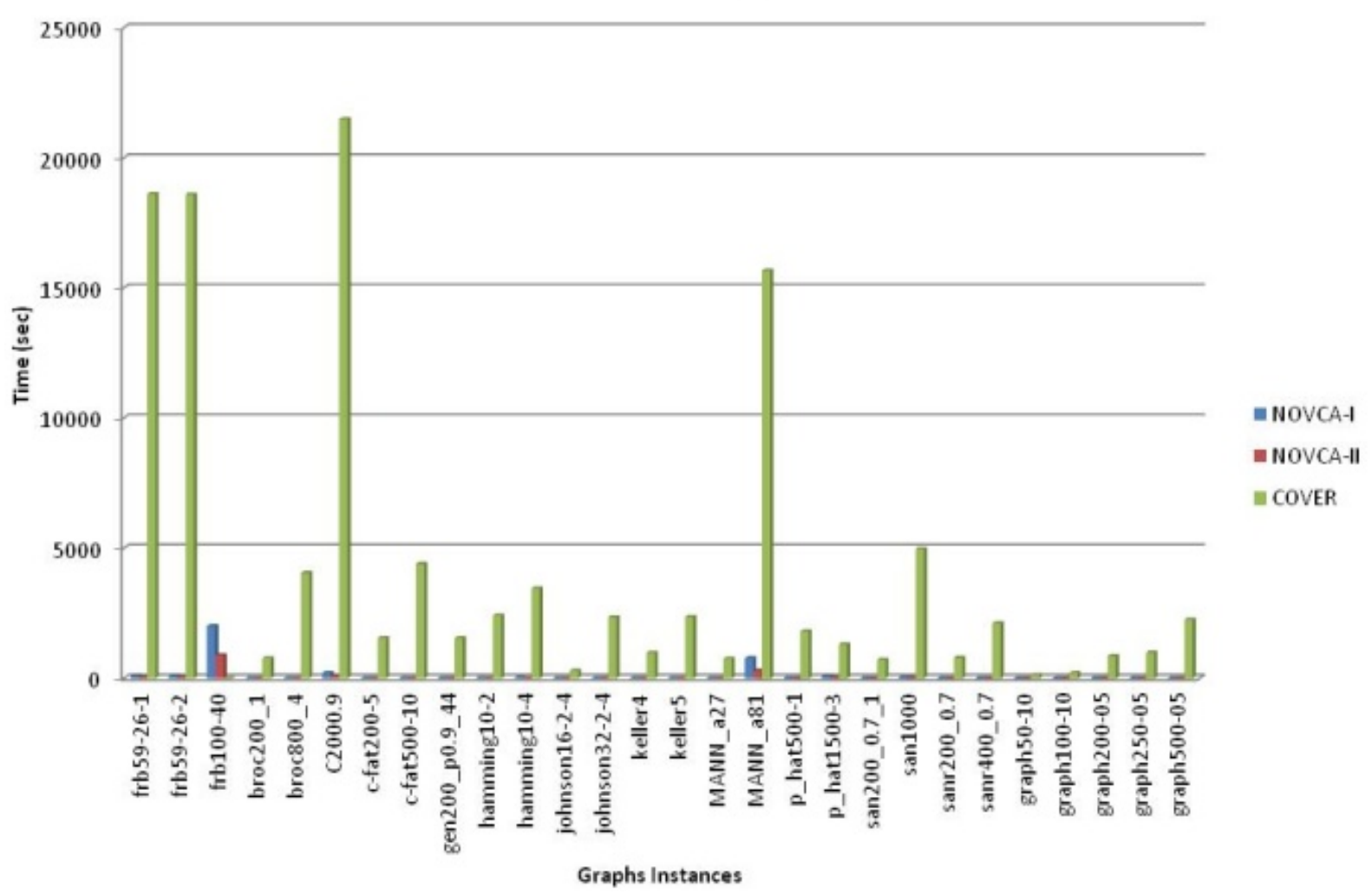

Fig. 4 Execution time for NOVCA-I, NOVCA-II, and COVER; no results from COVER for the instance frb100-40. 


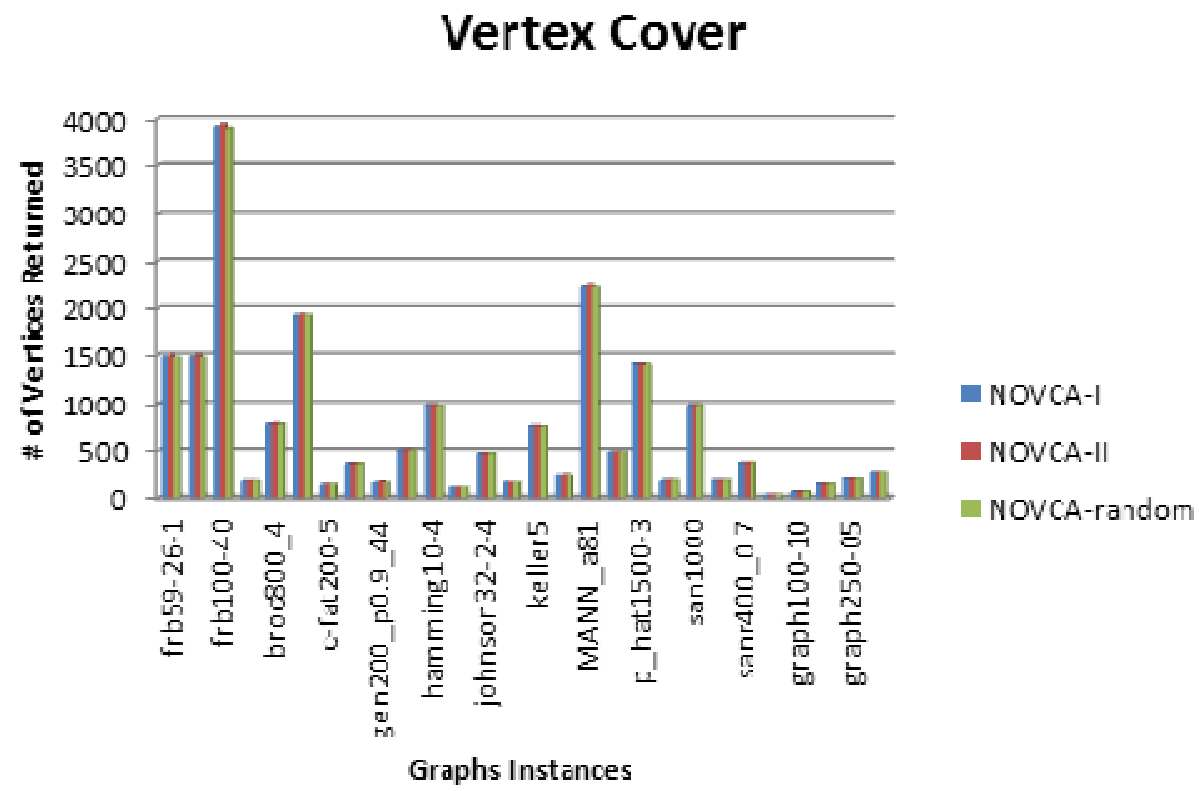

Fig. 5 Number of vertices returned by NOVCA-I, NOVCA-II, and NOVCA-random.

\section{Conclusions and Future Work}

All the versions of NOVCA algorithms, NOVCA-I, NOVCA-II, and NOVCA-random, provide optimal or near optimal vertex cover for known benchmark graphs. The experimental results depict that NOVCA is extremely fast compared to other available state-of-the-art MVC algorithms including COVER, PLS, and EWCC.

Future research will be focused in two areas: deriving a mathematical statement regarding the closeness of the approximation ratio to 1 , and investigating approaches to parallelizing the NOVCA algorithm.

\section{Acknowledgments}

This work made use of the High Performance Computing Resource in the Core Facility for Advanced Research Computing at Case Western Reserve University.

\section{References}

[1] Karp, R., 1972. "Reducibility among Combinatorial Problems." In Proceedings of a Symposium on the Complexity of Computer Computations, 85-103.

[2] Cormen, T., Leiserson, C., and Rivest, R. 2001. Introduction to Algorithms. United States :The MIT
Press.

[3] Bar-Yehuda, R., and Even, S. 1985. "A Local-Ratio Theorem for Approximating the Weighted Vertex Cover Problem." Annals of Discrete Mathematics 25: 27-45.

[4] Monien, B., and Speckenmeyer, E. 1985. "Ramsey Numbers and an Approximation Algorithm for the Vertex Cover Problem." ActaInformatica 22: 115-23.

[5] Halperin, E. 2000. 2002. "Improved Approximation Algorithms for the Vertex Cover Problem in Graphs and Hypergraphs.” SIAM J. on Computing 31 (5): 1608-23.

[6] Karakostas, G. 2005. "A Better Approximation Ratio for the Vertex Cover Problem." In Proceedings of the ICALP, 1043-50.

[7] Rudolph, G. 1998. "Finite Markov Chain Results in Evolutionary Computation." A tour d'horizon, Fundamenta Informaticae 35 (1-4): 67-89.

[8] Oliveto, P., He, J., and Yao, X. 2007. "Evolutionary Algorithms and the Vertex Cover Problem." In Proceedings of the IEEE Congress.

[9] Chen, J., Kanj, I., and Xia, G. 2005. Simplicity Is Beauty: Improved Upper Bounds for Vertex Cover. Technical report, Texas A\&M University.

[10] Abu-Khazm, F., Fellows, M., Langston, M., and Suters, W. 2007. Crown Structures for Vertex Cover Kernelization. Vol. 41. Egypt:Theory Comput. Systems, 411-30.

[11] Asgeirsson, E., and Stein, C. 2007. "Vertex Cover Approximation on Random Graphs." LNCS 4525: 285-96.

[12] Dinur, I., and Safra, S. 2001. The importance of being biased. Technical Report TR01-104, ECCC.

[13] Network Bench Node Degree, 2006. "http://nwb.slis.indiana.edu/" 
[14] Gajurel, S., and Bielefeld, R. 2012. "A Simple NOVCA: Near Optimal Vertex Cover Algorithm." Procedia Computer Science 9: 747-53.

[15] Gajurel, S., and Bielefeld, R. 2012. "A Fast near Optimal Vertex Cover Algorithm (NOVCA)." IJEA 3 (1): 9-18.

[16] Xu, K. 2012. "Vertex Cover Benchmark Instances (DIMACS \& BHOSLIB)." IJEA (international journal of Eexperimental algorithms) 3 (1): 1-18. Accessed October 25 ,

2012.

http://www.cs.hbg.psu.edu/benchmarks/vertex_cover.htm 1.
[17] Richter, S., Helmert, M., and Gretton, C. 2007. "A Stochastic Local Search Approach to Vertex Cover." In Proceedings of the 30th German Conference of Artificial Intelligence (KI), 412-26.

[18] Cai, S., Su, K., and Sattar, A. 2011. "Local Search with Edge Weighting and Configuration Checking Heuristics for Minimum Vertex Cover." Artifical Intelligence 175: 1672-96.

[19] Pullan, W. 2006. "Phased Local Search for the Maximum Clique Problem." Journal of Combinatorial Optimization 12: 303-23. 